\title{
Damage assessment in the stress field of scale, comparability and transferability
}

\author{
André Assmann ${ }^{1, a}$ and Stefan Jäger ${ }^{1}$ \\ ${ }^{1}$ geomer GmbH, Im Breitspiel 11B, 69126 Heidelberg, Germany
}

\begin{abstract}
Damage assessment is an important task requested in very different contexts. On the one hand very detailed studies are required for cost benefit analysis, on the other hand vulnerability and risk needs to be evaluated for political decisions and emergency situations (i.e. loss estimation of a large scale flood event). Even though a lot of studies have been performed and a lot of different methods are available by now, it is very difficult to use the results in the decision making process, as many methods are poorly described or are based on very special data, and therefore cannot be compared to each other. The attempt of regional (German federal state level) standardizations based on very detailed object based approaches are shown. Additionally, a European wide data set for asset mapping is described and a set of application examples is given. It is designed to enable users to compare the impact of different types of hazard. To take it one step further, a global map for vulnerability is presented that can be used to set up a monetary based data set if detailed statistical data is not available. Every type of data set and methodology has its special tasks to fulfil but there is also a high demand for harmonisation and comparability between different studies to enable better decision making. The next demand on data sets and methodologies is to perform time sequence analyses, therefore all data sets and methods presented fulfil the requirement of being updated regularly.
\end{abstract}

\section{Fields of application of damage assessment}

\subsection{Risk management}

Good risk management needs a lot of different information to support the decision making process. One important step in the process is to decide on the hot spots within the area of responsibility. Here the damage assessment comes in. Questions to be answered are: What total loss can be expected at a certain water level? What are critical thresholds?

In preparation of defense scenarios also the effectiveness of measures related to a certain scenario needs to be evaluated.

On the political level damage assessment is an important information tool for distributing budgets. Here again the effectiveness of budget spend compared to reduced losses is a major point influencing the decision.

\subsection{Cost benefit analysis}

A cost benefit analysis is a more detailed approach of performing estimations concerning the effectiveness of protection measures. While estimations done during an event are usually just related to this unique event, cost benefit analyses are oriented towards the lifetime of a measure. Taking the construction of a dike as an example, the costs of the dike and its maintenance should not be higher than the value of the houses to be protected. In the calculation also events higher than the level of protection need to be considered.

\section{Requirements concerning scale, comparability and transferability}

\subsection{Availability of data}

The availability and quality of data has a major impact on the methodology and quality of any assets map to be created. Usually there is a lot of data available, but not in a standardized way. Also the accessibility is very different; some communities provide well documented statistics while the same information is missing somewhere else. The same applies to land use data; sometimes the information is very detailed, including building information about age, usage and building material, in other regions only outdated settlement outlines are available. In these cases satellite data is of high interest.

Concerning data availability there are also some limitations due to privacy protection concerns. Also within governmental agencies there is a strong preference for using data from other governmental agencies and not to rely on data gathered from private institutions.

\footnotetext{
${ }^{\mathrm{a}}$ Corresponding author: $\underline{\text { aassm@geomer.de }}$
} 


\subsection{Comparability and transferability}

Even if the data situation is good, data sets are not directly comparable to each other due to different mapping standards. As a result of differing data situations different asset map products are produced which are not comparable to each other.

Studies in the frame of research projects most of the time include a lot of data gathering. This also applies to some very local cost benefit analysis. Such pioneering studies are very important concerning new knowledge gain. In most situations however, information like the exact level of the base floor, storey height, availability and use of cellars or even the building materials used are not available in the public digital data sets and often underlie the restrictions of data privacy protection. Due to these facts the results of these studies may be very beneficiary for the local usage but cannot be transferred to any other community or region not providing this kind of information. Even if there is enough budget available for data gathering, very detailed data cannot be collected for large regions within a short period of time. In the long term there is some hope that more data will be available in the future; but in the meantime one has to live with aggregated statistical data and has to handle the inhomogeneity of data sources.

\subsection{Damage functions}

If one is searching for damage functions, a whole lot of them for very detailed asset classes can be found (see figure 1).

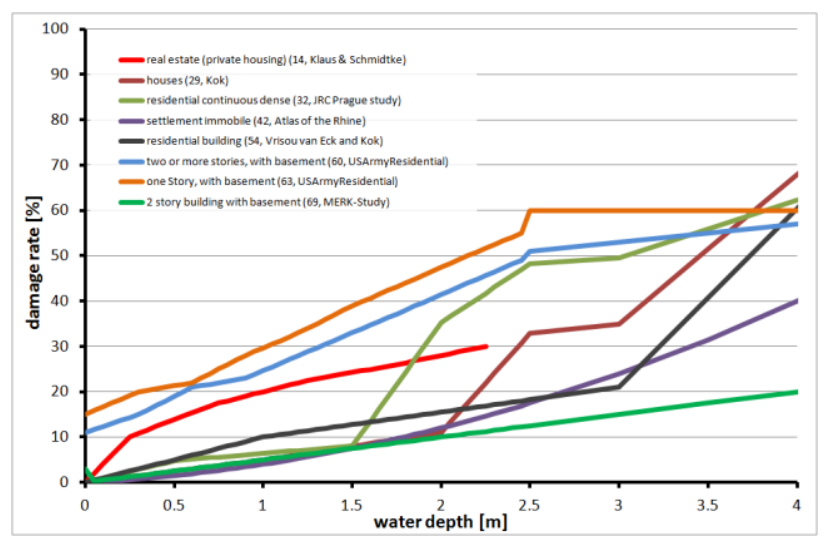

Figure 1. Comparison of different damage functions (private houses), even addressing the same class, the functions vary a lot due to the detailed definition, land use data set and data they are derived from

Quite often they are being used without any concern for the conditions under which they were constructed. For instance, many studies in Germany use the damage functions of the Rhine Atlas [1] and apply them to high resolution data sets, while the Rhine Atlas used CORINE Land Cover and therefore had to use some mixed land use classes. To give an example, the land use class "agricultural areas" included small settlements and farms in the asset calculation and therefore the assets are quite high compared to assets calculation differentiating the farm buildings from the fields. As the damage functions used in the Rhine Atlas are adjusted to the mixed asset of fields and agricultural buildings, transferring them to high resolution data sets means to make a big mistake.

Bearing this in mind, existing damage functions have to be checked and modified first, to adapt them to the planned usage. This requires to read and understand the assumptions made in the damage functions used (if such a description is available) and as well describe the assumptions made by oneself. As an example, the assumption that $70 \%$ of all cars can be removed from a certain area due to enough warning time limits the maximum value of a function to $30 \%$. This function has been used in the Danube FloodRisk project in the context of fluvial flooding (see figure 2). Using the same function for a flashflood prone area would lead to erroneous results because warning time is much shorter for flash flood events.

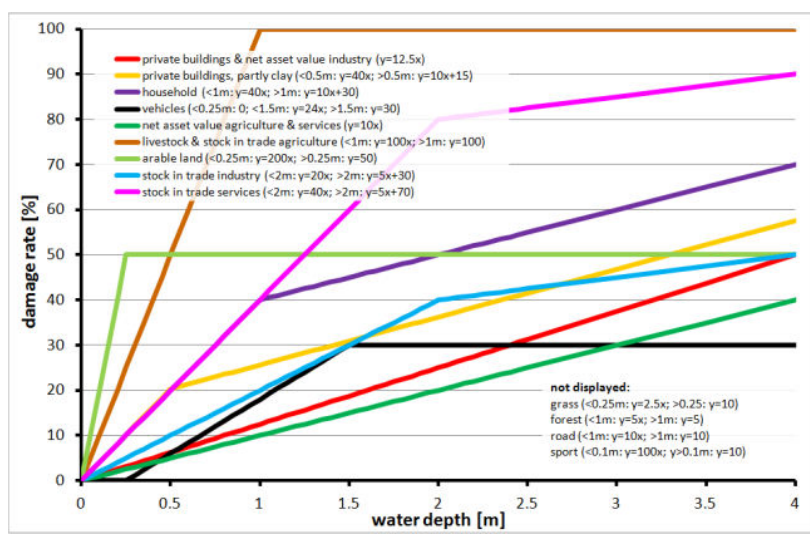

Figure 2. Damage functions being used for the Danube Atlas, the damage functions where derived from pre-existing ones and agreed on by an international expert group [2]

\section{Local to regional scale}

On local to regional scale object based approaches are favoured. In the context of flooding the focus is on buildings, where cadastral data is available. Having the building footprint, the usage of the building and sometimes even the height of the building, the asset totals acquired from national or federal statistics can be distributed to the single object. Due to the large amount of data and the complex data model, this is done by a well performing relational data base. Once such a data base model is implemented, aggregations using either interactive tools or outlines of statistical or political units can be performed within seconds.

The damage calculation itself is also performed using the database. Beforehand, buildings need to be intersected with the water depth information, for example classified by $5 \mathrm{~cm}$ contours. 


\section{Basic European Assets Map (BEAM)}

\subsection{Background and aims of BEAM}

In 1999 to 2001 the Atlas of the Rhine was created [1]. It was for the first time in Europe that consistent flood hazard and risk maps have been produced for a large river catchment covering several countries. For such a homogeneous approach the data available was limited and thus the European-wide CORINE land cover data set has been chosen, along with national statistics. The limitation of this approach was that it only addressed the flood topic and was not transferable to other types of natural hazards.

Lessons learned from the atlas project were also, that due to the limitations of the CORINE data set small settlements and industrial areas are leading to problems in disaggregating the statistical values. So if only very few or even no areas are covered (for example industrial areas) within a statistical unit the asset values are either too high for the few contained areas or the assets are not included at all.

As a successor of this work, the Basic European Assets Map (BEAM) was developed in the context of the EU-FP7 project SAFER [3] and further refined in the EU-FP7 project IncREO [4]. BEAM includes additional land use information using the HERE (former NAVTEQ) data set instead of the manual corrections made during the setting up of the Rhine Atlas. Also the road information has been taken from this source, providing the best homogenous quality all over Europe.

BEAM has been developed to estimate, evaluate and compare real and potential damages caused by natural disasters all across Europe, focussing both on European and national studies, which results in a significant advantage in cross-border areas.

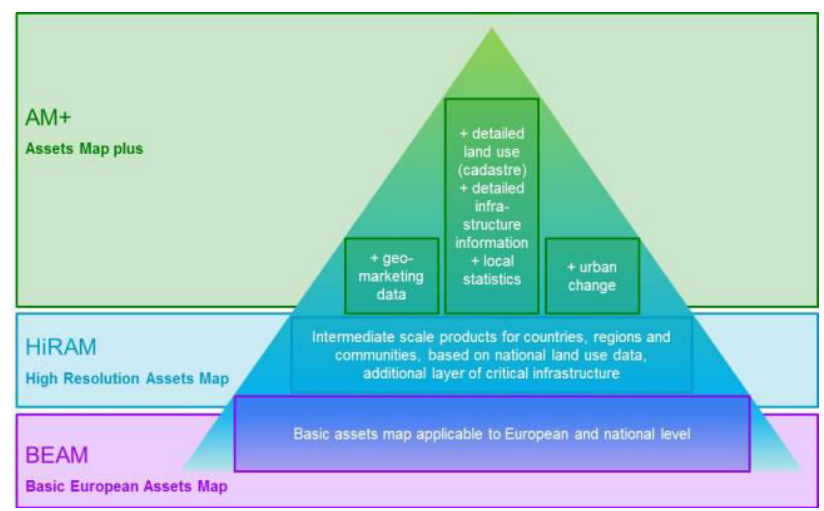

Figure 3. BEAM is meant to be part of a multi scale product concept, where each data set is compatible with the other ones

An additional advantage is the fact that the underlying methodology is scalable (see figure 3) and can be adapted to more detailed land use and statistical data if required. The fact that BEAM is coupled to the CORINE data set enables users to perform multi-temporal analyses using CORINE data from different years. As the concept strictly separates assets from damages, it can easily be applied to all types of risk and is thus an ideal basis for multi-risk-analysis.

BEAM displays assets in monetary values per area unit $\left(€ / \mathrm{m}^{2}\right)$. The complete asset information is composed of different layers, as within a damage assessment calculation each type of asset needs to be calculated applying a different damage function. The information provided is completed by an additional layer showing the population density.

\subsection{Setup of BEAM data set}

BEAM is composed of two major components: The asset volume taken from different statistical data bases and the land use data needed to disaggregate these values.

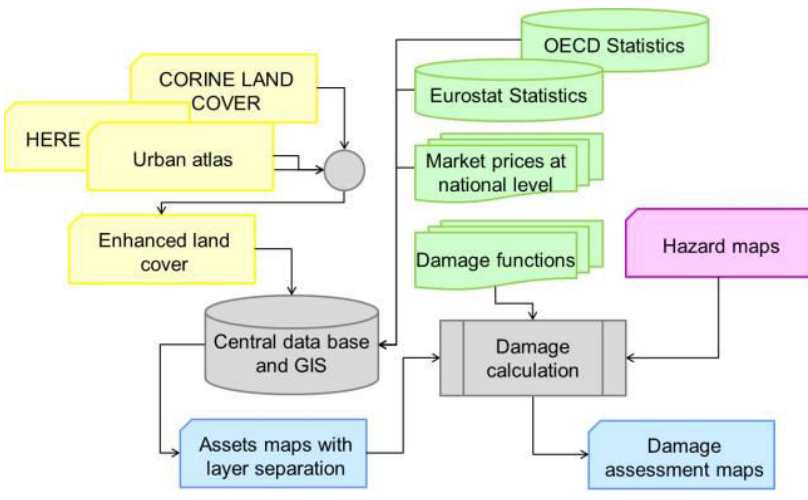

Figure 4. The BEAM central data base is feed by different European wide available data sets [5]

In some cases additional statistical data is needed to perform intermediate calculation steps. Details of this process are defined for each assets layer, an example is given in figure 5. As BEAM is using the net concept, the value decrease has to be taken into account. This is done by using a value decrease curve and the age composition of the vehicles. The approach for other assets follows a similar methodology.

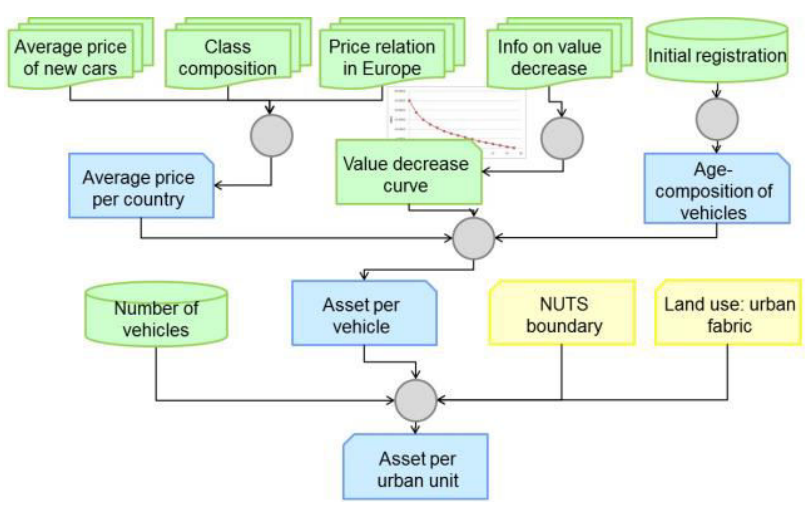

Figure 5. Example of a processing chain for one of the BEAM layers, in BEAM v2 the car asset is additionally disaggregated to road areas [5] 
The Basic European Assets Map consists of the following set of layers:

- Private housing: buildings and equipment (urban immobile assets)

- Household goods (urban mobile assets)

- Vehicle assets

- Industry: buildings and equipment (industrial net asset value, immobile)

- Industry: stock in trade (industrial mobile assets)

- Service and trade: buildings and equipment (service net asset value, immobile assets)

- Service and trade: stock in trade (service mobile assets)

- Agriculture: buildings and equipment (agricultural net asset value, immobile assets)

- Agriculture: stock in trade without livestock (agricultural mobile assets)

- Agriculture: livestock assets

Additionally, BEAM contains one combined layer that covers asset values for:

- Arable land

- Grassland

- Forest

- Road network

- Green urban areas and sport areas

In the updated version of 2012 (related to the CORINE Land Cover release in spring 2015) some refinement of the methodology has been performed:

- Integration of Urban Atlas data

- Integration of train lines

- Differentiation of agricultural land, grassland and forest based on soil and slope parameters

- Integration of urban infrastructure

- Consideration of moving vehicles in the asset distribution, this means that one part of the vehicle assets is allocated to the roads and the other to the settlement area

\subsection{Damage calculation}

Damage assessment can be conducted in two different ways: either by applying damage functions that provide a direct output of losses in monetary value, or by following a two-step approach which first calculates the assets and then applies damage functions that calculate the losses as a percentage.

BEAM resorts to the second approach. Thus, the underlying asset layers can be used for various types of hazards (e.g. in a multi risk analysis) and the damage functions can be applied to different regions. For example, the damage function for an average car can be the same in different countries or regions, although the age and value of an average car will probably differ.

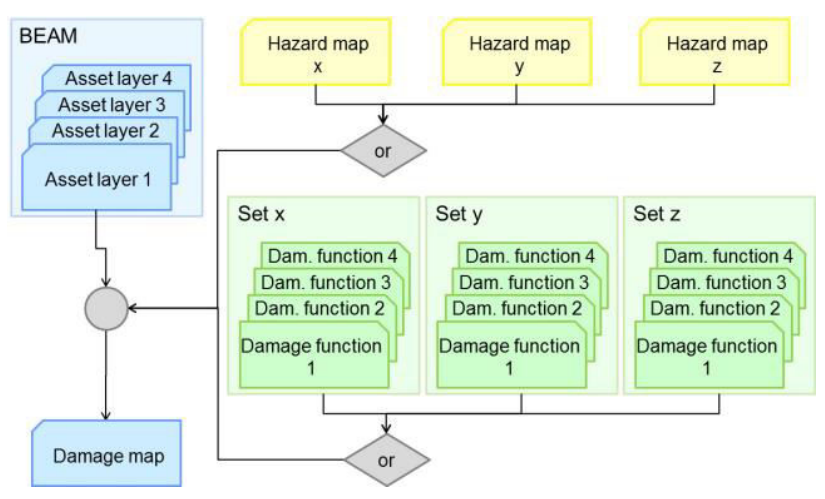

Figure 6. BEAM consists of several layers, each of them is addressed by a specific damage function, for each type of hazard a set of damage functions is needed

The damage functions applied are not part of the BEAM-product itself, but can be supplied to potential users from the various projects that have been using the BEAM dataset (see chapter 4.4 and figure 2) or they can be newly created for additional types of hazard on demand. Figure 6 illustrates the general workflow from damage calculations to the creation of hazard maps for different kinds of natural hazard.

\subsection{Fields of application}

As it is the idea of the BEAM dataset to be applied to different types of natural hazards and therewith to enable the comparison of their impact to a society, it has been tested and applied to a set of them.

- Flood: Danube and Elbe atlas, calculation of potential damage for different recurrence intervals $[2,5,6]$, for the Elbe the results correlate well with the damages of the different events in Saxony

- Windstorms: The Federal Office of Civil Protection and Disaster Assistance (Germany) performed a calculation to estimate the impact of windstorms

- Landslide: Test were performed in Buzau region in Romania within the IncREO project [4]

- Earthquake: Application tests were performed for Romania, using earthquake recordings of the last 100 years [4]

- Coastal flooding: Application tests were performed for Bulgaria, using storm surge modelling data run by Meteo France, the data was delivered, validated and used by the local Bulgarian Water Management Agency [4].

The different practical applications proved the usability of the product for different types of hazards.

The application in 12 European countries has made it somehow a standard in this domain. The data set created in the IncREO project is enhanced with some additional non-monetary information like elements at risk and population density. 


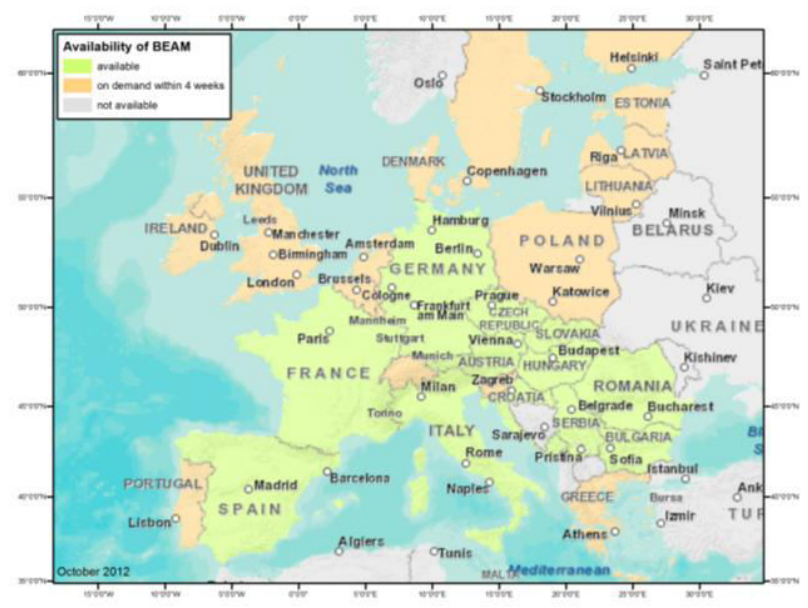

Figure 7. Availability of BEAM 2006 dataset, 12 countries are available on stock, for all countries where the CORINE land cover dataset is available BEAM can be produced on demand [3]

\section{Global Vulnerability Map}

The Global Resilience Map and the Global Vulnerability Map have also been designed and calculated within the frame of the IncREO project. Due to the fact that a lot of statistical data is not available on a global scale, a different approach was needed, to extend assets and damage estimations towards a global scale. Here the aim is not to do cost benefit analysis but to be able to do risk analysis and allocate hot spots.

The Global Resilience Map displays the resilience of the population with respect to natural hazards on a global scale. As there was no index available having a global coverage, this map was created by combining a set of different indices. Also, the map was not meant to show only a single value per country but to differentiate within the countries. To achieve this, the population density was used to break down these indices per country. Additional factors for disaggregating indices were derived by calculating the distance to airports and sea ports because they reflect the accessibility for national and international rescue forces in case of a disaster. The single indices used were:

- GNA final index, an index already combining different aspects of resilience

- Income per capita (US\$)

- Medical care (using number of doctors, medical staff and hospital beds)

- Education using the literacy as a measure

- Human rights index

- State stability index

Additionally, the gross national product (US\$) or the income per person has been used for the evaluation: A higher income usually supports a better equipment and availability of supplies.

Population density is another indicator to assess availability of infrastructure and equipment. In case of an event the needed equipment and infrastructure might not be available in sparsely inhabited areas, while in very densely populated areas the existing infrastructure might not suffice due to the high number of affected people. So there is an optimum in population density that is evaluated following the scheme in Figure 8.

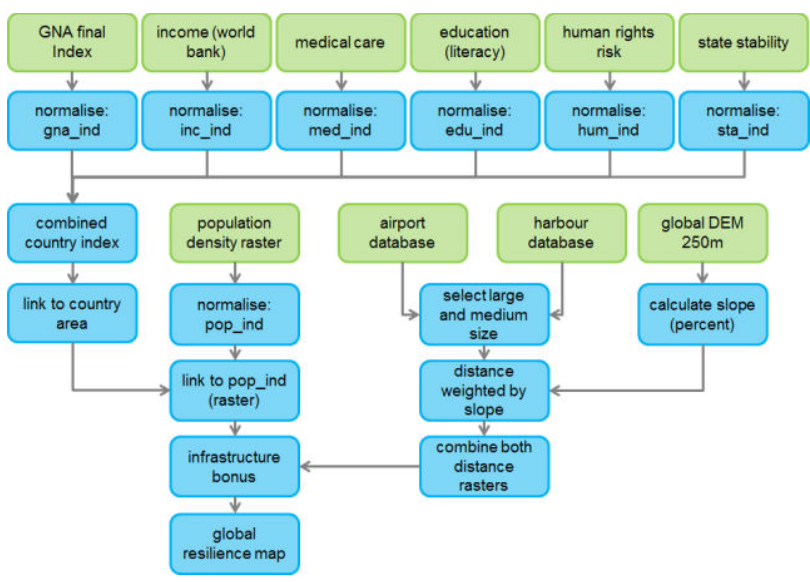

Figure 8. The Global Resilience Map uses a set of existing indices which are combined to a country index, additional information is used for disaggregation within the state territory [4]

All other inputs have been indices already in the source data bases and were only normalised.

Further assumptions used for producing the map are as follows:

- Only populated areas with a population density $>0.1$ person $/ \mathrm{km}^{2}$ are being considered vulnerable, so the focus of this map is only on human vulnerability.

- In case of an event, the population relies very much on external support. Especially airports and harbours are being used for transporting a large amount of emergency supplies in case of a natural disaster. A distance of $100 \mathrm{~km}$ seemed to be the outer limit of being of high relevance; the closer the distance, the better and faster support will be available. If the terrain is very rough, the accessibility towards these points will be much more difficult, this is covered by using the slope as a weighting factor in the calculation.

- Education is needed to know how to react in case of a disaster. As literacy is the basis for further education, it is a good and worldwide available measure to estimate the availability of basic knowledge.

- If in normal times human rights are respected and a state offers a high stability, the chances are much higher to manage a crisis situation. In contrast, if parts of the population are already discriminated in normal times, they will be at much higher risk of not getting the needed support in case of a natural disaster.

The processing of data is shown in in Figure 9. Marked in green you find the input data used in the calculation process, marked in blue are intermediate products and processes.

The resulting raster has values reaching from 1 to 5 where 1 is the lowest resilience and 5 the highest.

To derive the vulnerability using the concept of resilience described above, the population density is used as main impact factor for the vulnerability. So the more people live at a certain place, the higher the vulnerability. 


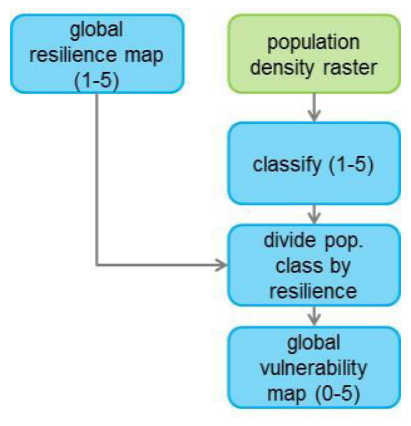

Figure 9. Scheme showing the generation of the Global Vulnerability Map from the Global Resilience Map [4]

To calculate the vulnerability, the value gained from the population reclassification is divided by the value of the global resilience map.

The resulting raster has values reaching from 0 to 5 where 0 is the lowest vulnerability and 5 the highest.

To obtain a result similar to a risk map, the vulnerability needs to be intersected with a hazard map. The values of the vulnerability index can be multiplied with a hazard index, resulting in a map containing some type of risk-zoning. Regarding the global scale there are usually some limitations concerning the hazard zoning, because most of the time there is limited information about intensity or recurrence intervals. Still such maps display hot spots very well and are a good source of information for internationally operating companies and organisations, especially ones working in disaster relief.

\section{Acknowledgements}

The research leading to these results has received funding from the European Community's Seventh Framework Programme (FP7/2007-2013) under grant agreement $\mathrm{n}^{\circ} 312461$ (Increasing Resilience through Earth Observation [IncREO]): www.increo-fp7.eu

\section{References}

1. ICPR (2001): Atlas of flood hazard and potential damage due to extreme floods of the Rhine. Published by the International Commission on the Protection of the Rhine (ICPR).

2. Danube FLOODRISK (2012): Danube-Atlas - Atlas of flood hazard and risk maps of the Danube. Published by the Ministry of Environment and Forests on behalf of Danube FLOODRISK, Bucharest, Romania.

3. SAFER (2012): http://www.copernicus.eu/projects/ safer (last access: $25^{\text {th }}$ Feb 2016)

4. IncREO (2014): www.increo-fp7.eu (last access: $25^{\text {th }}$ Feb 2016)

5. CESEP (2012). Manual of harmonized requirements on the flood mapping procedures for the Danube River - Data and Methods. Published by the Center for Environmental Sustainable Economic Policy (CESEP), Romania.

6. ELLA (2012). Elbe-Atlas - Preventive flood management measures by transnational planning (ELLA). $2^{\text {nd }}$ ed., published by the Saxon State Agency for Environment and Geology (LfUG), Dresden, Germany.



Figure 10. Printed example from the Global Vulnerability Map, the data set is available globally [4] 